\title{
BMJ Open Attaining a British consensus statement on managing idiopathic congenital talipes equinovarus (CTEV) through a Delphi process: a study protocol
}

\author{
Yael Gelfer (D) , ${ }^{1}$ Jose Blanco, ${ }^{2}$ Amanda Trees, ${ }^{3}$ Naomi Davis, ${ }^{4}$ \\ Rachel Buckingham, ${ }^{5}$ Anna C Peek, ${ }^{6}$ Elizabeth Wright, ${ }^{7}$ Rohan Rajan, ${ }^{8}$ \\ William Guy Atherton, ${ }^{9}$ Denise Watson, ${ }^{10}$ Vicky Easton, ${ }^{11}$ Neeraj Garg, ${ }^{12}$ \\ Jason Mavrotas, ${ }^{13}$ Sally Tennant, ${ }^{14}$ Tim Theologis (D) ${ }^{15}$
}

To cite: Gelfer Y, Blanco J, Trees A, et al. Attaining a British consensus statement on managing idiopathic congenital talipes equinovarus (CTEV) through a Delphi process: a study protocol. BMJ Open 2021;11:e049212. doi:10.1136/ bmjopen-2021-049212

- Prepublication history for this paper is available online. To view these files, please visit the journal online (http://dx.doi org/10.1136/bmjopen-2021049212).

Received 23 January 2021 Accepted 09 August 2021
Check for updates

(C) Author(s) (or their employer(s)) 2021. Re-use permitted under CC BY-NC. No commercial re-use. See rights and permissions. Published by BMJ.

For numbered affiliations see end of article.

Correspondence to

Dr Yael Gelfer;

yaelgelfer@gmail.com

\section{ABSTRACT}

Introduction Idiopathic congenital talipes equinovarus (CTEV) is the most common congenital limb deformity. Non-operative intervention using the Ponseti method has shown to be superior to soft tissue release and has become the gold standard for first-line treatment. However, numerous deviations from the Ponseti protocol are still reported following incomplete correction or deformity relapse. Significant variation in treatment protocols and management is evident in the literature. Reducing geographical treatment variation has been identified as one of The James Lind Alliance priorities in children's orthopaedics. For this reason, the British Society of Children's Orthopaedic Surgery (BSCOS) commissioned a consensus document to form a benchmark for practitioners and ensure consistent high quality care for children with CTEV.

Methods and analysis The consensus will follow an established Delphi approach aiming at gaining an agreement on the items to be included in the consensus statement for the management of primary idiopathic CTEV up to walking age. The process will include the following steps: (1) establishing a steering group, (2) steering group meetings, (3) a two-round Delphi survey aimed at BSCOS members, (4) final consensus meeting and (5) dissemination of the consensus statement. Degree of agreement for each item will be predetermined. Descriptive statistics will be used for analysis of the Delphi survey results.

Ethics and dissemination No patient involvement is required for this project. Informed consent will be assumed from participants taking part in the Delphi survey. Study findings will be published in an open access journal and presented at relevant national and international conferences. Charities and associations will be engaged to promote awareness of the consensus statement.

\section{INTRODUCTION}

Congenital talipes equinovarus (CTEV) is the most common congenital limb deformity, with an estimated incidence of 1-2 in 1000 live births. ${ }^{1-4}$ The Ponseti method has been
Strengths and limitations of this study

- Reduction of variation in practice has been identified as one of the top priorities in paediatric orthopaedic research and had motivated the formation of the British Society of Children's Orthopaedic Surgery (BSCOS) consensus groups.

- The steering group will include an expert panel of experienced, dedicated congenital talipes equinovarus practitioners.

- Meticulous application of the Delphi process.

- Support of BSCOS, which will facilitate participant recruitment for the steering group, the Delphi survey, dissemination and uptake of the consensus statement.

- The Delphi survey will be open to BSCOS members only resulting in a British Consensus document with non-consultant practitioners less well represented as BSCOS members.

shown to be an effective treatment for correction of deformity and in many healthcare settings has become the first-line treatment. ${ }^{5-9}$ However, there is significant variation in treatment protocols and outcome reporting, ${ }^{10} 11$ which has led some to resort to surgery for residual deformity and relapse, in rates of up to $53.3 \% .^{10}$ Examples of identified variations in treatment include age at the beginning of casting, the health professional involved in casting, casting technique, foot abduction brace regime and follow-up time. ${ }^{10} 11$

Patients with CTEV treated with soft tissue release have been reported to have poor longterm outcomes with a correlation between the extent of soft tissue release and functional impairment. $^{12-15}$

Previous attempts at developing consensus at European or national level included a relatively small number of experts and healthcare 
professionals. To our knowledge, there has been no previous attempt to develop a national consensus in clubfoot management involving all British specialists in paediatric orthopaedics. ${ }^{1617}$

The James Lind Alliance priority setting, which included clinicians as well as patients and parents, highlighted addressing variation in practice as one of the top 20 priorities. ${ }^{18}$ Geographic variation and lack of good evidence in multiple conditions such as management of developmental dysplasia of the hip and osteoarticular infection has motivated the British Society of Children's Orthopaedic Surgery (BSCOS) to develop consensus groups with primary CTEV management being one of them.

Consensus methods provide a mean of synthesising information and harnessing the insights of appropriate experts to enable decisions to be made. ${ }^{19}$

The Delphi technique is a structured process that uses a series of questionnaires or 'rounds' to gather information. Rounds are held until group consensus is reached. As a large number of individuals across diverse locations and areas of expertise can be included anonymously, this method is able to avoid domination of the consensus process by one or a few experts. ${ }^{20}$

It is therefore the aim of this study to describe a protocol for attaining a consensus document for the management of primary idiopathic CTEV from the time of diagnosis to walking age.

It is of the highest importance to ensure that the management of primary idiopathic CTEV follows the same practice and standard of care. Setting these standards will allow effective data collection and identify outliers, it will enable CTEV practitioners to share the published consensus document with carers and patient groups. To our knowledge, a standard of care document for the management of primary idiopathic clubfoot using the Ponseti technique according to the Delphi process does not exist in the literature.

\section{Scope}

The scope of the document will include the following five main areas:

(1) referral pathways and clinic setup; (2) initial assessment of patient and feet; (3) intervention, casting and tenotomy; (4) maintaining correction, the foot abduction brace and (5) early relapse. Accordingly, the proposed consensus document will serve as a standard that can be used to setup a CTEV service or as a benchmark for practitioners to improve their practice.

Owing to the variable presentation and challenging treatment as well as unpredictable outcomes, the approach to the management of non-idiopathic CTEV is beyond the scope of this consensus statement.

\section{METHODS AND ANALYSIS}

The consensus process will follow a recognised Delphi approach, ${ }^{20}{ }^{21}$ aiming at gaining agreement on the basic and important items to be included in the consensus statement for CTEV management. The stages and timeline are shown in figure 1.

\section{Establishing a steering group}

Members of the steering group are selected from BSCOS members and associate members, applicants being an orthopaedic consultant or any other CTEV practitioner including physiotherapist, nurse practitioner or plaster practitioner who are dedicated to clubfoot management. All applicants expressing an interest have submitted an expression of interest document and were subsequently selected by the BSCOS board. Owing to the COVID-19 pandemic all the consensus meetings are 'virtual' and are held using the 'zoom' video conferencing application. The aim of the steering group is to brainstorm and generate a list of suggested standards of practice to be scrutinised via a Delphi survey and facilitate convergence to a consensus opinion.

\section{The steering meetings}

The first steering meeting will nominate a Chair and a Secretary as well as deciding on the framework and the topics to be covered. Three virtual meetings, each lasting up to 3 hours, will cover topics deemed relevant by any member of the group. Each suggested topic will generate a process including a current literature review as well as the groups members' expert opinion. A fourth meeting will summarise all agreed statements to be presented to the BSCOS members in the Delphi survey. Every meeting will

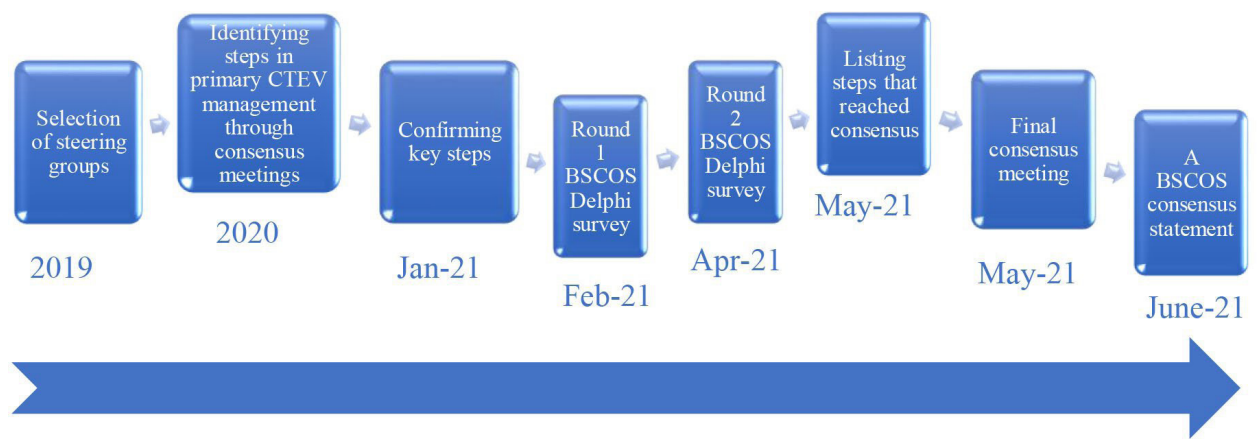

Figure 1 Flowchart of the study stages and timeline. BSCOS, British Society of Children's Orthopaedic Surgery; CTEV, congenital talipes equinovarus. 
generate minutes to be disseminated between all group members and available for all. The Chair will oversee the meetings, generate the agenda and manage any disagreement. The Secretary will generate the minutes and the steering group will finalise the list from round 1 to round 2. The steering group can split/amalgamate or modify questions between rounds 1 and 2 if they think that by doing so they can achieve better consensus. The steering group will also participate in the final consensus meeting (see below).

\section{The Delphi survey}

The Delphi survey will be pilot tested by the members of the steering committee to assess face and construct validity and acceptability. Practice information will be collected at the start of the Delphi survey and each respondent will be provided with a unique identifier enabling personalised reminders for completion of subsequent rounds, while maintaining anonymity to the steering group. This information will include position, years of experience and whether CTEV is a part of their main practice. If a participant declares that they don't manage clubfoot and don't feel qualified to respond they would have the option to terminate the survey at that point. Participants will be asked to score each outcome in the survey using a Grading of Recommendations Assessment, Development and Evaluation (GRADE) scale, which ranges from 1 to 9 (1-3=not important, 4-6=important but not critical and $7-9=$ critical for inclusion). ${ }^{22}$ There will be free-text fields to allow the participants to give a reason for their decision and/or any additional topics that they consider to be important and should be considered to be included in the consensus document. The surveying process will be conducted by one external and one internal researcher, who are not members of the steering group, certifying the methodology throughout the study

\section{Round 1}

The generated list of items is circulated to BSCOS members to assess the 'degree of agreement'. Round 1 will be open to the panel for 6 weeks and reminder emails will be sent at 2 week intervals before it is deemed complete. The responses will be summarised and reported anonymously. Items scored between 7 and 9 (critical importance) by $\geq 75 \%$ of the participants will be directly moved to the consensus meeting. Items scored between 1 and 3 (not important) by $\geq 25 \%$ will be excluded. All other items, including those scored between 4 and 6 (important but not critical) will be deemed as 'no consensus' and will be carried out in round 2 of the Delphi survey. Any further suggestions will be added to the generated list for the subsequent round when appropriate.

\section{Round 2}

During round 2 of the Delphi survey, participants will be asked to re-score the importance of each item that was scored as no consensus in the first round. The scoring process will be carried out similar to that in round 1 with 4 weeks between the two rounds.

The responders will be able to see the overall scores from round 1 of each item they will be asked to re-score.

\section{Analysis}

Items from both rounds will be analysed descriptively: the number of participants rating each item from rounds 1 and 2 will be calculated. Consensus will be defined as shown below. All items in 'no consensus' from the round 1 will be presented in the round 2 for rating. All items in 'consensus in', 'consensus out' and 'no consensus' categories will be presented in the final consensus meeting for discussion.

- Consensus in: $\geq 75 \%$ participants scored it as "critical for inclusion' and $<25 \%$ of participants scored it as 'notimportant for inclusion.

- Consensus out: $\geq 75 \%$ participants scored it as 'notimportant for inclusion' and $<25 \%$ of participants scored it as 'critical for inclusion'.

- No consensus: Anything else not included in the other two categories.

The SPSS software will be used to calculate the median and IQR for each item. These will be particularly relevant in gaining further insight on the level of agreement for each individual statement. Rather than a simple agree/ disagree outcome, the median and IQR offer additional detail that will assist the steering group in processing the 'no consensus' statements between the two rounds and in reaching decisions on the consensus statements at the final meeting.

Missing scores will be taken into account. The denominator for each Delphi survey item will be the number of participants completing that item, rather than the number of participants completing the Delphi survey overall (ie, a participant may choose not to score a particular Delphi item for whatever reason). This will be taken into account both in the descriptive statistics and in the calculation of the median and IQR. The number of participants who register to the survey but do not fill in the questionnaire (eg, because of lack of expertise on the subject) will be recorded but not taken into account for the analysis.

The JISC survey software will be used for the Delphi survey. ${ }^{23}$ The online interface will initially present a summary of the project and questions on respondent's profile. It will also include instructions on how to score each question/statement. The software will automatically generate reminders and will allow participants to save and complete their responses at a later stage if they wish, as long as this is within the timeframe of the survey. After completion of each round of the survey, all data will be extracted as a simple CSV file so that it can be easily imported into the analysis software. The data extracts are (1) user data, (2) scores data and (3) missing data. A consensus report will provide a summary of participant's scores across both rounds which will be available for the final consensus meeting. 


\section{The final consensus meeting}

The final consensus meeting will be hosted for the purpose of finalising the consensus document. The meeting will include all previous steering group members as well as any additional member the steering group feels might bring additional value or expertise. Remote access to the meeting will be available as per previous meetings. A report including the results from the two-round survey will be available before the meeting.

\section{Final decisions}

At the meeting, Delphi survey results will serve as the basis for the discussion and development of the final consensus document.

Any item categorised as 'consensus in' will be proposed to be included in the final document, while any item categorised as 'consensus out' will be excluded. The panel members will vote to accept the list of items or suggest items that warrant further discussion. The voting system will be anonymous, using an online platform. ${ }^{24}$ Items that are categorised as 'no consensus' will be discussed individually. The final consensus document will be agreed on by the steering group. A second meeting will be arranged in the event of no agreement on the final consensus document.

\section{The consensus statement}

The final output of this process will be a set of recommendations for best practice management of primary idiopathic CTEV up to walking age in the UK. As a standard of clinical management, it will not involve other stakeholders such as patients and families in the process. The statement will provide a British standard of care document involving BSCOS members.

\section{Patient and public involvement}

No patients involved.

\section{DISCUSSION}

The geographic variability of practice and documentation has been identified as a research priority in The James Lind Alliance priority setting by clinicians, patients and parents. ${ }^{18}$ This has resulted in the formation of several BSCOS consensus groups aiming to improve standard of care and documentation.

An European consensus meeting was set up in 2012 in Stockholm to define standards for Ponseti treatment. Clubfoot experts from 12 countries met to discuss goals, standards and challenges based on the literature review and personal experience. ${ }^{16}$ The outcome document intended to form a blueprint for orthopaedic societies and policymakers to formulate national guidelines. A paper aiming to provide a foundation for standardisation of clubfoot treatment in the Netherlands was published in $2017 .{ }^{17}$ The most important clinical question addressed concerned the primary treatment of clubfoot with a clear recommendation of the Ponseti method as the optimal method of primary clubfoot treatment. The outcome document was a collaboration between the Dutch parents' association and an expert panel of six paediatric orthopaedic surgeons and provided guidelines of implementation of the Ponseti method as the first-line standard of care treatment for CTEV. ${ }^{17}$ This is the first study on the development of a British consensus statement regarding the best standard of care in primary idiopathic CTEV up to walking age. The advantages of this study are the sound methodology including a thorough literature review for each item, a selection of a panel of experienced, knowledgeable and dedicated CTEV practitioners and the meticulous application of the Delphi process, involving the whole society of paediatric orthopaedic surgeons and not just the experts. The limitations of the study is that non-consultant practitioners are less well represented as BSCOS members with the Delphi survey aiming at BSCOS members only.

As per previously published standard of care guidelines, ${ }^{25}$ this document might have limitations when used in a different country. An international consensus statement is currently beyond the scope of this project. An international statement with experts and society members recruited from other countries and specialist societies following the same protocol would be an achievable next step. It would be interesting to assess how comparable these statements will be. Furthermore, our protocol can be modified for use in conditions other than idiopathic CTEV. In particular, future BSCOS consensus projects in other paediatric orthopaedic conditions are likely to follow a similar protocol.

In conclusion, this study is expected to develop a national consensus document on the management of primary idiopathic CTEV. It is likely that this document will serve as benchmark for the treatment of the condition nationally and will encourage consistent management of the condition. Moreover, the document is likely to be used for auditing individual units and practices for the purpose of governance and appraisal.

\section{ETHICS AND DISSEMINATION}

Informed consent will be assumed by all participants taking part in the Delphi survey. Participants (BSCOS members) will be approached via the Society's Webmaster via email. All members have provided General Data Protection Regulation compliant consent to be contacted by the society for matters relevant to the profession, including surveys.

Support of societies, associations and charities that represent health professionals will facilitate dissemination of the consensus statement and subsequent uptake, for example BSCOS and the British Orthopaedic Association. A one-page summary will be provided to the clinicians and families.

The findings will be submitted for publication in peer-reviewed and open-access journals and will be presented at national and international conferences on CTEV. TheAGREE APPRAISAL OF GUIDELINES FOR RESEARCH \& EVALUATION) checklist will be used as the principal for result reporting. Journals and funding bodies 
will be approached to promote awareness of the consensus document.

\section{Author affiliations}

${ }^{1}$ Trauma and Orthopaedic Department, St George's Hospital, London, UK

${ }^{2}$ Paediatric Orthopaedics, North West Anglia NHS Foundation Trust, Peterborough,

UK

${ }^{3}$ Physiotherapy, James Cook University Hospital, Middlesbrough, UK

${ }^{4}$ Paediatric Orthopaedic Surgery, Manchester University NHS Foundation Trust,

Manchester, UK

${ }^{5}$ Paediatric Orthopaedics, Oxford University Hospitals NHS Foundation Trust, Oxford, UK

${ }^{6}$ Paediatric Orthopaedics, University Hospitals of Leicester NHS Trust, Leicester, UK

${ }^{7}$ Orthopaedics, Southampton Children's Hospital, Southampton, UK

${ }^{8}$ Orthopaedics, University Hospitals of Derby and Burton NHS Foundation Trust,

Derby, UK

${ }^{9}$ Trauma and Orthopaedics, Bristol Royal Hospital for Children, Bristol, UK

${ }^{10}$ Physiotherapy, Chelsea and Westminster Hospital NHS Foundation Trust, London,

UK

${ }^{11}$ Paediatric Physiotherapy, Norfolk and Norwich University Hospitals NHS

Foundation Trust, Norwich, UK

${ }^{12}$ Orthopaedics, Alder Hey Children's Hospital, Liverpool, UK

${ }^{13}$ Core Surgical Trainee, Newcastle Upon Tyne Hospitals NHS Foundation Trust,

Newcastle Upon Tyne, UK

${ }^{14}$ Paediatric Orthopaedics, Royal National Orthopaedic Hospital NHS Trust, London, UK

${ }^{15}$ Paediatric Orthopaedic Surgery, Oxford University Hospitals NHS Foundation Trust, Oxford, UK

\section{Twitter Yael Gelfer @yaelgelfer and Tim Theologis @Tim Theologis}

Contributors YG: Developed the structure and details of the Delphi survey items, drafted the manuscript, approved the final version before submission. JB: Developed the structure and details of the Delphi survey items, refined the manuscript, critically reviewed the manuscript and approved the final version before submission. AT, ND: Developed the structure and details of the Delphi survey items, developed the on line survey and data collection for the analysis, critically reviewed the manuscript and approved the final version before submission. RB, ACP, EW, RR, WGA, DW, VE, NG: Developed the structure and details of the Delphi survey items, critically reviewed the manuscript and approved the final version before submission. JM: Developed the on line survey and data collection for the analysis, critically reviewed the manuscript and approved the final version before submission. ST: Developed the structure and details of the Delphi survey items, developed the on line survey and data collection for the analysis, responsible for the management of the study and is the chair of the steering committee, critically reviewed the manuscript and approved the final version before submission. TT: Developed the structure and details of the Delphi survey items, developed the on line survey and data collection for the analysis, introduced the idea of the consensus groups and protocol, provided supervision and input to all aspects of the study, critically reviewed the manuscript and approved the final version before submission. The structure and details of the Delphi survey items were developed by the British CTEV consensus group. The manuscript was drafted by YG and was refined by JB, AT, ND, ST and TT. JM developed the online survey and data collection for the analysis. ST is responsible for the management of the study and is the chair of the steering committee. TT introduced the idea of the consensus groups and protocol, provided supervision and input to all aspects of the study. All authors critically reviewed the manuscript and approved the final version before submission.

Funding The authors have not declared a specific grant for this research from any funding agency in the public, commercial or not-for-profit sectors.

\section{Competing interests None declared.}

Patient and public involvement Patients and/or the public were not involved in the design, or conduct, or reporting, or dissemination plans of this research.

Patient consent for publication Not required.

Provenance and peer review Not commissioned; externally peer reviewed.

Open access This is an open access article distributed in accordance with the Creative Commons Attribution Non Commercial (CC BY-NC 4.0) license, which permits others to distribute, remix, adapt, build upon this work non-commercially, and license their derivative works on different terms, provided the original work is properly cited, appropriate credit is given, any changes made indicated, and the use is non-commercial. See: http://creativecommons.org/licenses/by-nc/4.0/.

ORCID iDs

Yael Gelfer http://orcid.org/0000-0001-8212-9999

Tim Theologis http://orcid.org/0000-0002-4758-9081

\section{REFERENCES}

1 Gibbons PJ, Gray K. Update on clubfoot. J Paediatr Child Health 2013;49:E434-7.

2 Dobbs MB, Gurnett CA. Update on clubfoot: etiology and treatment. Clin Orthop Relat Res 2009;467:1146-53.

3 Smythe T, Kuper H, Macleod D, et al. Birth prevalence of congenital talipes equinovarus in low- and middle-income countries: a systematic review and meta-analysis. Trop Med Int Health 2017;22:269-85.

4 Chen C, Kaushal N, Scher DM, et al. Clubfoot etiology: a metaanalysis and systematic review of observational and randomized trials. J Pediatr Orthop 2018;38:e462-9.

5 Morcuende JA, Dolan LA, Dietz FR, et al. Radical reduction in the rate of extensive corrective surgery for clubfoot using the Ponseti method. Pediatrics 2004;113:376-80.

6 Jaqueto PA, Martins GS, Mennucci FS, et al. Functional and clinical results achieved in congenital clubfoot patients treated by Ponseti's technique. Rev Bras Ortop 2016;51:657-61.

7 Dunkley M, Gelfer Y, Jackson D, et al. Mid-Term results of a physiotherapist-led Ponseti service for the management of nonidiopathic and idiopathic clubfoot. J Child Orthop 2015;9:183-9.

8 Herzenberg JE, Radler C, Bor N. Ponseti versus traditional methods of casting for idiopathic clubfoot. J Pediatr Orthop 2002;22:517-21.

9 Cooper DM, Dietz FR. Treatment of idiopathic clubfoot. A thirty-year follow-up note. J Bone Joint Surg Am 1995;77:1477-89.

10 Gelfer Y, Wientroub S, Hughes K, et al. Congenital talipes equinovarus: a systematic review of relapse as a primary outcome of the Ponseti method. Bone Joint J 2019;101-B:639-45.

11 Gelfer Y, Hughes KP, Fontalis A, et al. A systematic review of reported outcomes following Ponseti correction of idiopathic club foot. Bone Jt Open 2020;1:457-64.

12 Laaveg SJ, Ponseti IV. Long-term results of treatment of congenital club foot. J Bone Joint Surg Am 1980;62:23-31.

13 Dobbs MB, Nunley R, Schoenecker PL. Long-term follow-up of patients with clubfeet treated with extensive soft-tissue release. J Bone Joint Surg Am 2006;88:986-96.

14 Ippolito E, Farsetti P, Caterini R, et al. Long-term comparative results in patients with congenital clubfoot treated with two different protocols. J Bone Joint Surg Am 2003;85:1286-94.

15 Smith PA, Kuo KN, Graf AN, et al. Long-term results of comprehensive clubfoot release versus the Ponseti method: which is better? Clin Orthop Relat Res 2014;472:1281-90.

16 Böhm S, Sinclair M. Report of the 1st European consensus meeting on Ponseti clubfoot treatment: Karolinska Institutet Stockholm, July 6th 2012. J Child Orthop 2013;7:251-4.

17 Besselaar AT, Sakkers RJB, Schuppers HA, et al. Guideline on the diagnosis and treatment of primary idiopathic clubfoot. Acta Orthop 2017;88:305-9.

18 Vella-Baldacchino M, Perry DC, Roposch A, et al. Research priorities in children requiring elective surgery for conditions affecting the lower limbs: a James Lind alliance priority setting partnership. BMJ Open 2019;9:e033233.

19 Jones J, Hunter D. Qualitative research: consensus methods for medical and health services research. BMJ1995;311:376-80.

20 Boulkedid R, Abdoul H, Loustau M, et al. Using and reporting the Delphi method for selecting healthcare quality indicators: a systematic review. PLoS One 2011;6:e20476.

21 Murphy MK, Black NA, Lamping DL, et al. Consensus development methods, and their use in clinical Guideline development. Health Technol Assess 1998;2:i-iv, 1-88.

22 Guyatt GH, Oxman AD, Kunz R, et al. Grade guidelines: 2. Framing the question and deciding on important outcomes. J Clin Epidemiol 2011;64:395-400.

23 University of Oxford. IT help. Available: https://help-archive.it.ox.ac. uk

24 VoxVote free and easy mobile voting tool for any speaker or teacher. Available: https://www.voxvote.com/

25 British Orthopaedic Association. BOA standards for trauma and orthopaedics (BOASTs), 2008. Available: https://www.boa.ac.uk/ standards-guidance/boasts.html 